\title{
Gas Sensing Properties of Novel CuO Nanowire Devices
}

\author{
S. Steinhauer ${ }^{1}$, E.Brunet ${ }^{1}$, T. Maier ${ }^{1}$, G. C. Mutinati ${ }^{1}$, A. Köck ${ }^{1}$, O. Freudenberg ${ }^{2}$ \\ ${ }^{1}$ AlT Austrian Institute of Technology $\mathrm{GmbH}$, Vienna, Austria \\ stephan.steinhauer.fl@ait.ac.at \\ ${ }^{2}$ Siemens AG, Corporate Technology, Corporate Research and Technologies, Munich, Germany
}

\begin{abstract}
:
We report on novel gas sensing devices which are realized by the on-chip synthesis of cupric oxide $(\mathrm{CuO})$ nanowires during the thermal oxidation of electroplated copper microstructures. By this method, it is possible to fabricate conductometric gas sensors with an electrical resistance dominated by a multitude of suspended $\mathrm{CuO}$ nanowires resulting in a huge sensor surface area. For these devices, resistance changes during exposure to different oxygen concentrations, to relative humidity and to small concentrations of the toxic gas carbon monoxide (CO) are investigated. Due to the promising sensing results and the CMOS backend compatibility of the fabrication process steps, the presented devices may find applications in integrated gas sensing devices.
\end{abstract}

Key words: $\mathrm{CuO}$ nanowire, on-chip synthesis, gas sensor, $\mathrm{CO}$ detection

\section{Introduction}

There is a strongly increasing demand for highly sensitive gas detecting devices in numerous applications ranging from personal safety devices to industrial process control. Metal oxide based gas sensors, which rely on changes of electrical conductance due to the interaction with the surrounding gas, have been thoroughly investigated in the past decades. A most powerful strategy to improve sensor performance is the implementation of nanowires as sensing elements, which have a high surface to volume ratio and thus a strong interaction between the surrounding gas and the material.

Various metal oxides have been extensively studied for applications in conductometric gas sensors [1]. Among them, the p-type conducting, narrow band gap material $\mathrm{CuO}$ has been investigated in thin film [2] and thick film [3] devices. Especially $\mathrm{CuO}$ nanowires have attracted much scientific attention as these nanostructures have also been proposed for several other applications such as photovoltaics, field emitting devices or catalysts [4]. CuO nanowires can be synthesized by wetchemical methods [5], templating methods [6], electrospinning [7] or thermal oxidation of copper [8]. Several groups have reported on the gas sensing properties of $\mathrm{CuO}$ nanowires [9-11] and showed their high sensitivity to different target gases. However, the fabrication of conventional nanowire devices most commonly involves a nanowire transfer process step from the synthesis substrate to the device substrate, which in most cases leads to poor reproducibility and low fabrication yield. As an alternative to nanowire printing or dielectrophoresis based assembly processes we use thermal oxidation of electroplated copper structures in order to synthesize $\mathrm{CuO}$ nanowires directly on-chip and are therefore able to integrate them in a gas sensing device.

\section{Device Fabrication}

The $\mathrm{CuO}$ nanowire devices are fabricated as follows: A Ti/Cu seed layer $(50 \mathrm{~nm}$ and $150 \mathrm{~nm}$, respectively) is sputter-deposited onto silicon substrates covered with $500 \mathrm{~nm}$ of thermal oxide. Afterwards, a photolithographic process defines the areas for an electroplating process, which is applied to selectively deposit a $2.5 \mu \mathrm{m}$ thick Cu layer. Next, the photoresist is removed and the residual $\mathrm{Ti} / \mathrm{Cu}$ seed layer is wet-etched using sodium persulfate and a diluted 5\% hydrofluoric acid solution. Contact pads are structured utilizing a lift-off process of a sputtered Ti/Au layer $(5 \mathrm{~nm}$ and $200 \mathrm{~nm}$ thickness, respectively). After an ion milling process for surface cleaning, the samples are oxidized in a furnace under a constant flow of pure oxygen at $400^{\circ} \mathrm{C}$ for three hours, which results in the growth of $\mathrm{CuO}$ nanowires. A typical device is shown in Fig. 1: eight oxidized copper lines with a width of $25 \mu \mathrm{m}$ and a gap of $5 \mu \mathrm{m}$ in between (before thermal oxidation) are contacted by two opposing Ti/Au contact pads. During the thermal oxidation process the initial gap distance is first of all considerably reduced 
to around $2.5 \mu \mathrm{m}$ due to oxide growth; moreover the gap is bridged by suspended $\mathrm{CuO}$ nanowires which form an electrical contact between the oxidized copper lines (see inset of Fig.1) and thus between the Ti/Au contact pads. Typically, nanowires grown with this specific technology show dimensions with lengths in the range of few $\mu \mathrm{m}$ and diameters between $15 \mathrm{~nm}$ and $70 \mathrm{~nm}$.

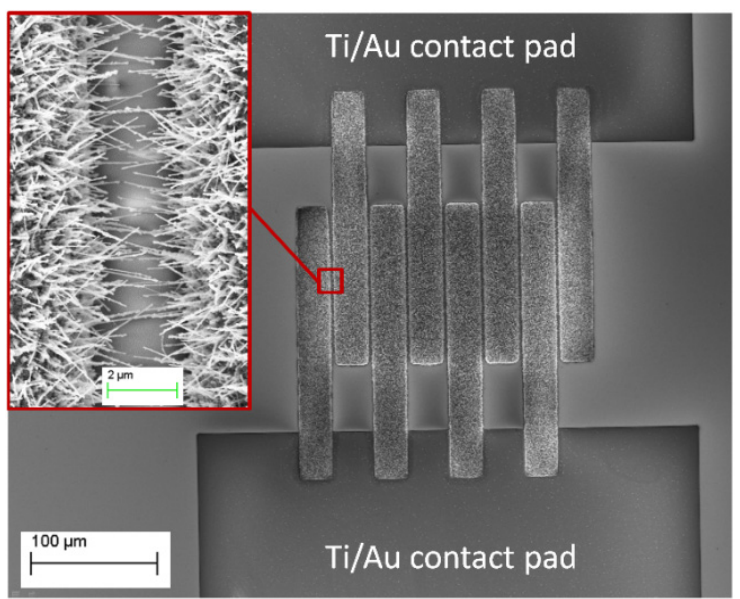

Fig. 1. Gas sensing device consisting of eight oxidized copper lines and $\mathrm{CuO}$ nanowires bridging the gap in between. Device resistance is measured between the upper and the lower Ti/Au contact pad and is dominated by the suspended $\mathrm{CuO}$ nanowires.

Specific test structures on the same chip are used in order to evaluate the series resistance of the oxidized copper lines. Their contribution to the overall resistance is several orders of magnitude lower than the resistance of the $\mathrm{CuO}$ nanowires that bridge the gap between the oxidized copper lines. From this we conclude that the gas sensitivity can be unambiguously attributed to the resistance change of the multitude of suspended $\mathrm{CuO}$ nanowires.

\section{Gas Measurements}

As conductometric gas sensors have to be operated at elevated temperatures, the sensor chips are glued to a silicon carrier together with commercially available microheaters (10x2 Pt6,8-0,4, Delta-R GmbH) and a Pt100 temperature sensor $(4 \times 1$ Pt100A, Delta-R $\mathrm{GmbH}$ ), which are soldered to a ceramic mounting (Fig.2). Electrical connections to the sensor chip are established by wedge bonding of a $25 \mu \mathrm{m}$ thick Au wire. The sensors are tested in an automated gas measurement setup, where background gas, target gas concentration, relative humidity and sensor operation temperature can be precisely controlled. Measurements are carried out under a constant gas flow of $1000 \mathrm{sccm}$ at a fixed operation temperature. The electrical resistance of the sensor devices is measured by a Keithley
2400 Source Meter in constant current operation.

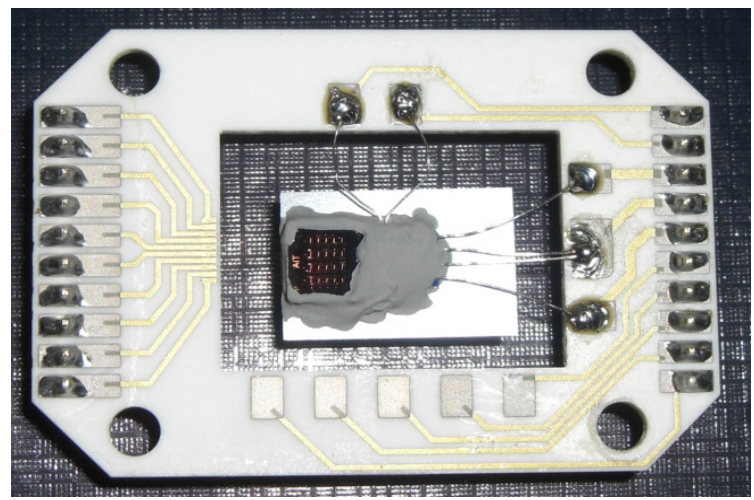

Fig. 2. Sensor chip glued to a suspended silicon carrier together with two microheaters (soldered leads on the right) and a Pt100 (soldered leads on top). The electrical connections from the $\mathrm{CuO}$ nanowire devices to the ceramic mounting are realized by wedge bonding to the Au lines on the left.

As pre-adsorbed oxygen plays a crucial role in the detection of water vapor and reducing gases, the influence of oxygen on the sensor resistance is investigated. For this purpose, the sensor is operated at $\mathrm{T}=300^{\circ} \mathrm{C}$ under a constant flow of nitrogen with oxygen concentrations varying from $5 \%$ to $25 \%$. During the oxygen exposure, the sensor resistance decreases sharply to around $25 \%$ of its initial value (Fig.3). This sensing behavior is consistent with literature models, which will be discussed later on. The large sensor signals show that the resistance of the $\mathrm{CuO}$ nanowire devices is effectively influenced by the surrounding gas atmosphere.

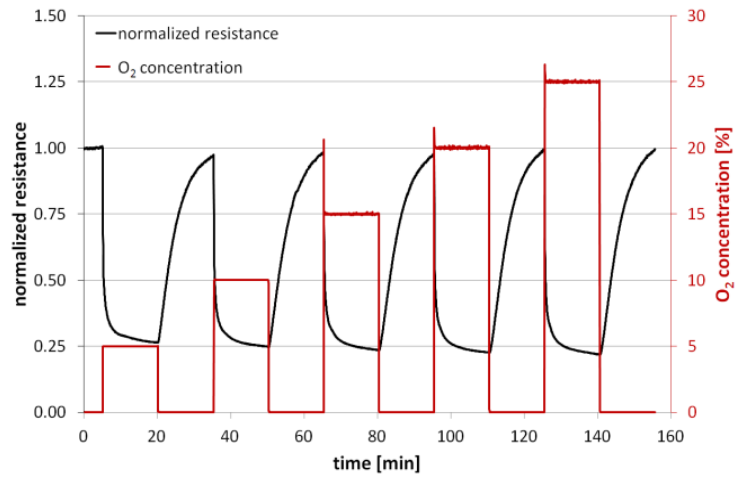

Fig. 3. Normalized $\mathrm{CuO}$ nanowire device resistance (black line) at an operation temperature of $T=300^{\circ} \mathrm{C}$ in nitrogen with oxygen pulses (red line) at different concentrations.

As water vapor is present in almost all practical sensing applications, the sensor response to humidity has to be investigated. The $\mathrm{CuO}$ nanowire device is operated at a temperature of $\mathrm{T}=300^{\circ} \mathrm{C}$ under a constant flow of synthetic air with pulses of different concentrations of humidity. The sensor resistance increases 
considerably during the exposure to water vapor up to nearly three times of its initial value, but does not fully recover again in dry synthetic air (Fig.4).

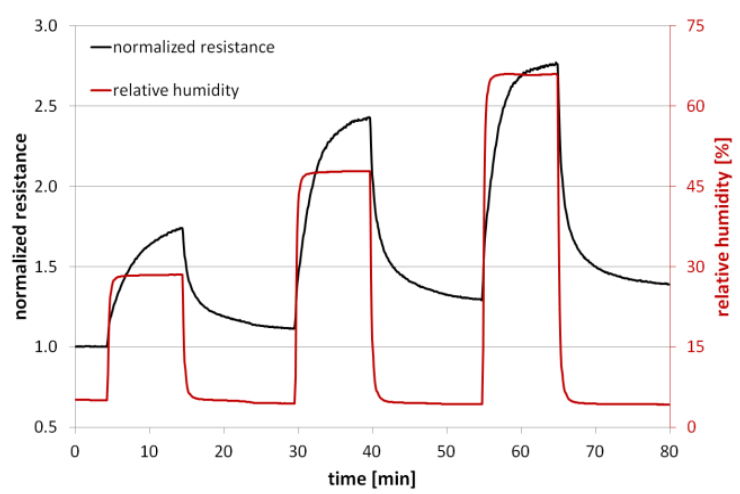

Fig. 4. Normalized $\mathrm{CuO}$ nanowire device resistance (black line) at an operation temperature of $T=300^{\circ} \mathrm{C}$ in synthetic air with humidity pulses (red line) at different concentrations.

Furthermore, the sensitivity of the $\mathrm{CuO}$ nanowire devices towards small concentrations of the toxic gas $\mathrm{CO}$ is investigated. Again, an operation temperature of $\mathrm{T}=300^{\circ} \mathrm{C}$ and dry synthetic air as background gas is chosen. $\mathrm{CO}$ concentrations of $10 \mathrm{ppm}$, 50ppm and 100ppm are detected with corresponding signal changes of $4 \%, 14 \%$ and $20 \%$, respectively. Compared to the humidity measurement, the time constants for detection and sensor recovery are considerably smaller. Furthermore, the CuO nanowire device fully recovers in pure synthetic air, which is not the case after the exposure to water vapor.

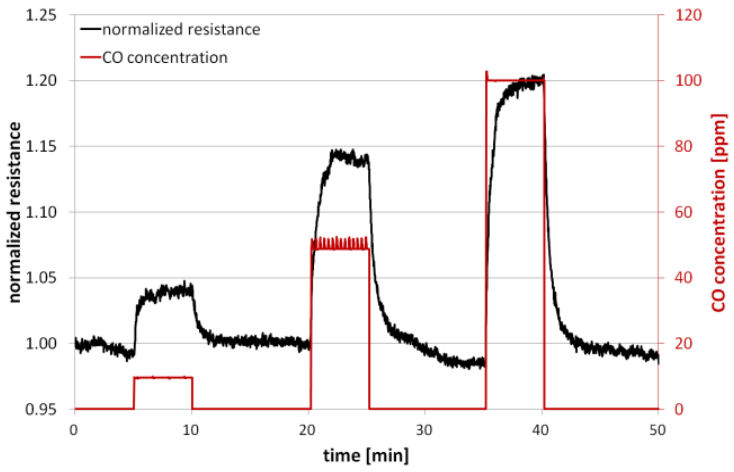

Fig. 5. Normalized CuO nanowire device resistance (black line) at an operation temperature of $T=300^{\circ} \mathrm{C}$ in synthetic air with CO pulses (red line) at different concentrations.

\section{Discussion}

When comparing the gas measurements of the presented $\mathrm{CuO}$ nanowire sensor with literature results, the differences and similarities to conventional devices have to be discussed. Single or multiple nanowires between two metal electrodes rely on the gas sensitivity of the conducting channel [4] or on the modulation of a Schottky barrier to a metal contact [12]. However, in our case the contact resistance between touching nanowires has to be taken into account due to the sensor morphology. Therefore, gas-induced resistance changes are attributed to different carrier concentrations in the nanowire channels as well as modulation of the potential barrier at the nanowire-nanowire contacts. The latter effect should be very well comparable to the sensing mechanism of thick film devices that consist of touching grain boundaries.

The reaction of oxygen with an adsorption site results in ionosorbed oxygen species $\mathrm{O}^{-}$and the generation of a hole, which leads to an accumulation layer at the surface of the p-type conducting $\mathrm{CuO}$ nanowire [13]. The common model for the detection of reducing gases is based on the decrease of oxygen species at the surface leading to an increase of resistance, which was observed when the $\mathrm{CuO}$ nanowire sensor was exposed to CO (Fig.4). The measurement of different oxygen concentrations in nitrogen (Fig.3) shows that the effect of ionosorbed oxygen accounts for a $75 \%$ resistance decrease. It can be assumed that this is the theoretical maximum of the sensor response in the presence of reducing gases for the $\mathrm{CuO}$ nanowire device in the presented configuration. The sensing mechanism for water vapor was explained by the decrease of ionosorbed oxygen and the formation of $\mathrm{OH}^{-}$hydroxyl groups leading to an increase of resistance and a change of the electronic affinity of the material [3]. In our measurements, we observe different sensor response times during exposure to humidity and $\mathrm{CO}$, which shows the different kinetics of the chemical reactions. Moreover, the incomplete sensor recovery could be an indication for remaining hydroxyl groups at the surface. However, further measurements at different operation temperatures, varying humidity concentrations and longer time scales are needed in order to get a better understanding of the sensor behavior during and after water vapor exposure.

\section{Summary}

We have investigated the gas sensing properties of novel $\mathrm{CuO}$ nanowire devices and report on the sensitivity to different oxygen concentrations, relative humidity and $\mathrm{CO}$. The results show the excellent sensing performance and can be explained by literature models for the gas sensitivity of p-type metal oxide semiconductors. Furthermore, the use of a multitude of fully suspended $\mathrm{CuO}$ nanowires turned out to be a very well-suited gas sensing configuration. 
The main advantage of the presented devices is the simple fabrication process, which allows the synthesis of $\mathrm{CuO}$ nanowires at well defined locations directly on the device chip. Therefore, no additional nanowire transfer step is needed and the device properties can be easily tailored by changing the dimensions and the distance between the copper structures. As the process temperatures during nanowire synthesis do not exceed $400^{\circ} \mathrm{C}$, the presented concept may be used in a CMOS backend process, which enables the fabrication of fully integrated gas sensing devices.

\section{Acknowledgements}

The authors are grateful to $\mathrm{H}$. Homolka from the Institute of Sensor and Actuator Systems, Vienna University of Technology, for preparation of the ceramic sensor mountings. This work was partly funded by the FFG Austrian Research Promotion Agency within the MNT-Eranet project "NanoSmart" (No. 828691) and the WWTF Vienna Science and Technology Fund within the project "Mathematics and Nanosensors" (No. MA09028).

\section{References}

[1] G. Korotcenkov, Metal oxides for solid-state gas sensors: What determines our choice?, Materials Science and Engineering B 139, 1-23 (2007); doi:10.1016/j.mseb.2007.01.044

[2] N. S. Ramgir et al., Sub-ppm $\mathrm{H}_{2} \mathrm{~S}$ sensing at room temperature using $\mathrm{CuO}$ thin films, Sensors and Actuators B: Chemical 151, 90-96 (2010); doi: 10.1016/j.snb.2010.09.043

[3] M. Hübner et al., Influence of humidity on $\mathrm{CO}$ sensing with p-type $\mathrm{CuO}$ thick film gas sensors, Sensors and Actuators B: Chemical 153, 347-353 (2011); doi: 10.1016/j.snb.2010.10.046

[4] L. Liao et al., Multifunctional $\mathrm{CuO}$ nanowire devices: p-type field effect transistors and $\mathrm{CO}$ gas sensors, Nanotechnology 20, 085203 (2009); doi: $10.1088 / 0957-4484 / 20 / 8 / 085203$

[5] W. Wang et al., A simple wet-chemical synthesis and characterization of $\mathrm{CuO}$ nanorods, Applied Physics A 76, 417-420 (2003); doi: 10.1007/s00339-002-1514-5

[6] Y. Dongliang et al., Preparation and characterization of $\mathrm{CuO}$ nanowire arrays, Journal of Semiconductors 30, 072003 (2009); doi: 10.1088/1674-4926/30/7/072003

[7] H. Wu et al., Fabrication, assembly, and electrical characterization of $\mathrm{CuO}$ nanofibers, Applied Physics Letters 89, 133125 (2006); doi: 10.1063/1.2355474

[8] X. Jiang et al., CuO Nanowires Can Be Synthesized by Heating Copper Substrates in Air, Nano Letters 2, 1333-1338 (2002); doi: $10.1021 / \mathrm{nl} 0257519$

[9] Y.-S. Kim et al., CuO nanowire gas sensors for air quality control in automotive cabin, Sensors and Actuators B: Chemical 135, 298-303 (2008); doi:10.1016/j.snb.2008.08.026

[10] D. Li et al., Conductometric chemical sensor based on individual $\mathrm{CuO}$ nanowires, Nanotechnology 21, 485502 (2010); doi: 10.1088/0957-4484/21/48/485502

[11] B. J. Hansen et al., Transport, Analyte Detection, and Opto-Electronic Response of p-Type $\mathrm{CuO}$ Nanowires, Journal of Physical Chemistry C 114, 2440-2447 (2010); doi: 10.1021/jp908850j

[12] Y. Hu et al., Supersensitive, Fast-Response Nanowire Sensors by Using Schottky Contacts, Advanced Materials 22, 3327-3332 (2010); doi: 10.1002/adma.201000278

[13] N. Barsan et al., Modeling of sensing and transduction for p-type semiconducting metal oxide based gas sensors, Journal of Electroceramics 25, 11-19 (2010); doi: $10.1007 / \mathrm{s} 10832-009-9583-\mathrm{x}$ 